\title{
Absolute Temperature Field Measurements in Flames based on Infrared Thermo- Spectroscopic Imaging
}

\author{
by A. AOUALI*, S. CHEVALIER*, A. SOMMIER*, J.C. BATSALE* and C. PRADERE*
}

* I2M TREFLE, UMR CNRS-UB-ENSAM 5295, Esplanade des Arts et Métiers, 33405 Talence Cedex, France

\begin{abstract}
Plasma torches are a type of source that generates very high temperatures. They are mainly used in surface treatment applications and ultimate waste treatment. The knowledge of the temperature field in plasma torches is of prime interest for researchers and industry to model and optimize the torch design. First experiments to measure the plasma torch temperature were reported by J.-L. Gardarein et al. [1] using a probe with a thermocouple directly inserted into the plasma. This first measurement led to the knowledge of plasma heat flux, but it was an intrusive method which gives only a single average value. To move forward, researches at I2M are conducted in collaboration with Europlasma to thermally and chemically characterize plasma torches with the end goal of 3D non-contact heat flux and temperature field measurements.
\end{abstract}

To ensure the previous objective, radiometric techniques are developed which provide adequate means to measure contactless temperature [2]. This temperature can be deduced from the luminance measured using an IR camera if the emissivity of the body is known [3]. In flame measurements, the assumption of thermal equilibrium is generally done [4] which makes the cartography of the emissivity, $\varepsilon$, to be equal to the absorptivity, $\alpha$. Thus, by measuring the transmissivity, $\tau$, of an IR beam through the flame, one can deduce the absorptivity, where $\alpha=1-\tau$ in semi-transparent medium, and therefore obtain $\varepsilon$. This methodology is validated at the lab scale using a small flame generated by a burner, an IR source and camera.

In Figure 1, the experimental setup to measure the flame temperature is depicted. Three simultaneous acquisitions are made using a shutter synchronized with the camera: (1) the IR source beam alone, (2) the IR source beam + the proper emission of the flame, and (3) the proper emission of the flame alone. From this 3-images method, the total hemispheric transmissivity $\tau$ is measured. This result is presented is Figure 2(a) where the thermo-dependence of the transmittivity in the flame is observed. The transmittivity ranges from 0.16 to 0.4 , which leads to an emissivity range between 1 and 0.84 (Figure 2(b)). As expected, the flame differs from the black body (emissivity of 1) which has justified the need of emissivity measurements to obtain the true flame temperature from an IR camera. This thermodependency of emissivity is expected to be strengthened in plasma torches which reach $5000 \mathrm{~K}$ (about 4-5 time hotter than the flame used in this experiments). In the oral presentation, this 3-images methodology will be presented in details and results of flame temperature measurements will be reported.

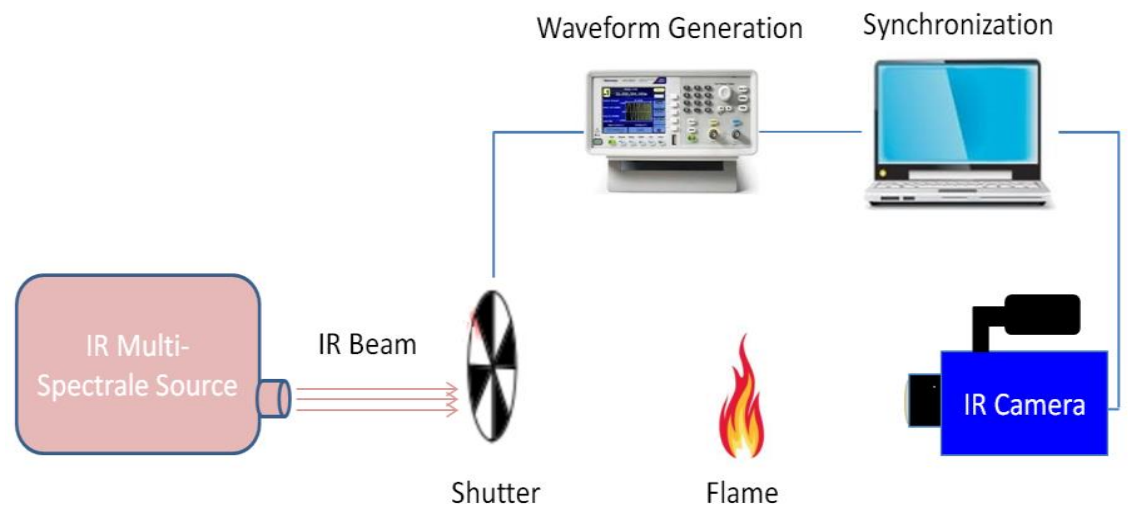

Fig. 1. Set-up used for the 3-images method 


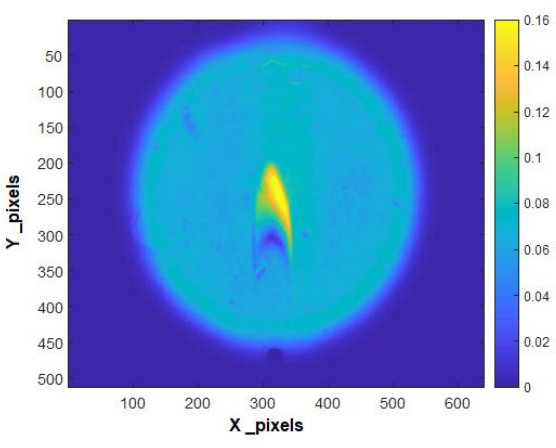

(a)



(b)

Fig. 2. (a). Cartography of Transmissivity, (b). Cartography of Emissivity

\section{REFERENCES}

[1] J-Laurent Gardareina Jean-Luc Battaglia Stefan Lohle Pierre Jullien Bruno Van Ootegem Jacques Couzi Jean-Pierre Lasserre., "Miniaturized heat flux sensor for high enthalpy plasma flow characterization". Inverse Problems in Science and Engineering, vol. 21, 2013.

[2] M. Romano M. Ryu J. Morikawa J. Batsale and C. Pradere., "Simultaneous microscopic measurements of thermal and spectroscopic fields of a phase change material", Infrared Physics and Technology. 2016.

[3] H. Philippe J. Cedelle I. Negreanu. "Infrared Technique for Simultaneous Determination of Temperature and Emissivity", Infrared Physics and Technology, vol. 55, 2012.

[4] André MOUTET, Jean CRABOL, Lionel NADAUD., "Température des gaz et des flames". Techniques de l'ingénieur, 1974. 